Open Access

\title{
Impact of arrhythmia on diagnostic performance of adenosine stress CMR in patients with suspected or known coronary artery disease
}

\author{
Simon Greulich, Hannah Steubing, Stefan Birkmeier, Stefan Grün, Kerstin Bentz, Udo Sechtem \\ and Heiko Mahrholdt ${ }^{*}$
}

\begin{abstract}
Background: The diagnostic performance of adenosine stress cardiovascular magnetic resonance (CMR) in patients with arrhythmias presenting for work-up of suspected or known CAD is largely unknown, since most CMR studies currently available exclude arrhythmic patients from analysis fearing gating problems, or other artifacts will impair image quality. The primary aim of our study was to evaluate the diagnostic performance of adenosine stress CMR for detection of significant coronary stenosis in patients with arrhythmia presenting for 1) work-up of suspected coronary artery disease (CAD), or 2) work-up of ischemia in known CAD.

Methods: Patients with arrhythmia referred for work-up of suspected CAD or work-up of ischemia in known CAD undergoing adenosine stress CMR were included if they had coronary angiography within four weeks of CMR.

Results: One hundred fifty-nine patients were included ( $n=64$ atrial fibrillation, $n=87$ frequent ventricular extrasystoles, $n=8$ frequent supraventricular extrasystoles). Of these, $n=72$ had suspected CAD, and $n=87$ had known CAD. Diagnostic accuracy of the adenosine stress CMR for detection of significant CAD was $73 \%$ for the entire population (sensitivity $72 \%$, specificity $76 \%$ ). Diagnostic accuracy was $75 \%$ (sensitivity $80 \%$, specificity $74 \%$ ) in patients with suspected CAD, and $74 \%$ (sensitivity $71 \%$, specificity $79 \%$ ) in the group with known CAD. For different types of arrhythmia, diagnostic accuracy of CMR was $70 \%$ in the atrial fibrillation group, and $79 \%$ in patients with ventricular extrasystoles. On a per coronary territory analysis, diagnostic accuracy of CMR was $77 \%$ for stenosis of the left and $82 \%$ for stenosis of the right coronary artery.

Conclusion: The present data demonstrates good diagnostic performance of adenosine stress CMR for detection of significant coronary stenosis in patients with arrhythmia presenting for work-up of suspected CAD, or work-up of ischemia in known CAD. This holds true for a per patient, as well as for a per coronary territory analysis.
\end{abstract}

Keywords: CMR, Adenosine stress, Arrhythmia, Coronary artery disease, Risk stratification

\footnotetext{
* Correspondence: Heiko.Mahrholdt@rbk.de

Department of Cardiology, Robert Bosch Medical Center, Auerbachstrasse

110, 70376 Stuttgart, Germany
}

\section{() Biomed Central}

(C) 2015 Greulich et al. Open Access This article is distributed under the terms of the Creative Commons Attribution 4.0 International License (http://creativecommons.org/licenses/by/4.0/, which permits unrestricted use, distribution, and reproduction in any medium, provided you give appropriate credit to the original author(s) and the source, provide a link to the Creative Commons license, and indicate if changes were made. The Creative Commons Public Domain Dedication waiver (http://creativecommons.org/publicdomain/zero/1.0/) applies to the data made available in this article, unless otherwise stated. 


\section{Background}

Coronary artery disease (CAD) is the leading cause of death in the western world [1]. Current clinical practice guidelines recommend noninvasive stress testing for 1) work-up of suspected CAD [2], and 2) work-up of ischemia in known CAD [3].

Adenosine stress cardiovascular magnetic resonance (CMR) is a noninvasive stress-testing modality offering high diagnostic accuracy without need for radiation or acoustic window [4-7]. However, up to $90 \%$ of patients with CAD suffer from frequent ventricular ectopic beats [8], and/or other arrhythmias [9, 10]. Unfortunately, the diagnostic performance of adenosine stress CMR in this important subgroup is widely unknown, since most CMR studies currently available exclude arrhythmic patients from analysis [6,11-13] fearing gating problems, or other artifacts will impair image quality [14].

Thus, our primary aim was to evaluate the diagnostic performance of adenosine stress CMR for detection of significant coronary stenosis in patients with arrhythmia presenting for 1) work-up of suspected CAD, or 2) work-up of ischemia in known CAD. In addition, we aimed to assess the diagnostic performance of adenosine stress CMR for different types of arrhythmia and for different coronary territories.

\section{Methods}

\section{Patient population}

All patients referred for 1) work-up of suspected CAD, and 2) work-up of ischemia in known CAD undergoing adenosine stress CMR at our institution between January 2011 and June 2014 were prospectively screened for study enrolment on a consecutive basis. We included all patients with arrhythmia during the stress CMR procedure (see definition of arrhythmia below), who underwent invasive coronary angiography within 4 weeks before or after the stress CMR, and who gave written informed consent to the protocol, which was approved by the local institutional review board (University of Tübingen, Germany). Exclusion criteria for the analysis were collateralized total occlusions revealed by coronary angiography, since in those cases no ischemia may be present despite occlusion, nondiagnostic images due to breathing artifacts, withdrawal of consent before completion of procedure, or other technical problems (Fig. 1). Baseline characteristics of the patient population can be viewed in Table 1.

\section{Definitions}

Relevant coronary stenosis/CAD was defined as $\geq 70 \%$ narrowing of the luminal diameter in at least one projection of at least one major epicardial artery, or $\geq 50 \%$ narrowing of the left main [2].

Suspected CAD: Patients without prior history of CAD.

Known CAD: Patients with prior myocardial infarction and/or revascularization procedure(s) such as percutaneous coronary intervention (PCI) or coronary artery bypass graft (CABG).

CAD-type late gadolinium enhancement (LGE): Subendocardial or transmural LGE consistent with prior myocardial infarction [15].

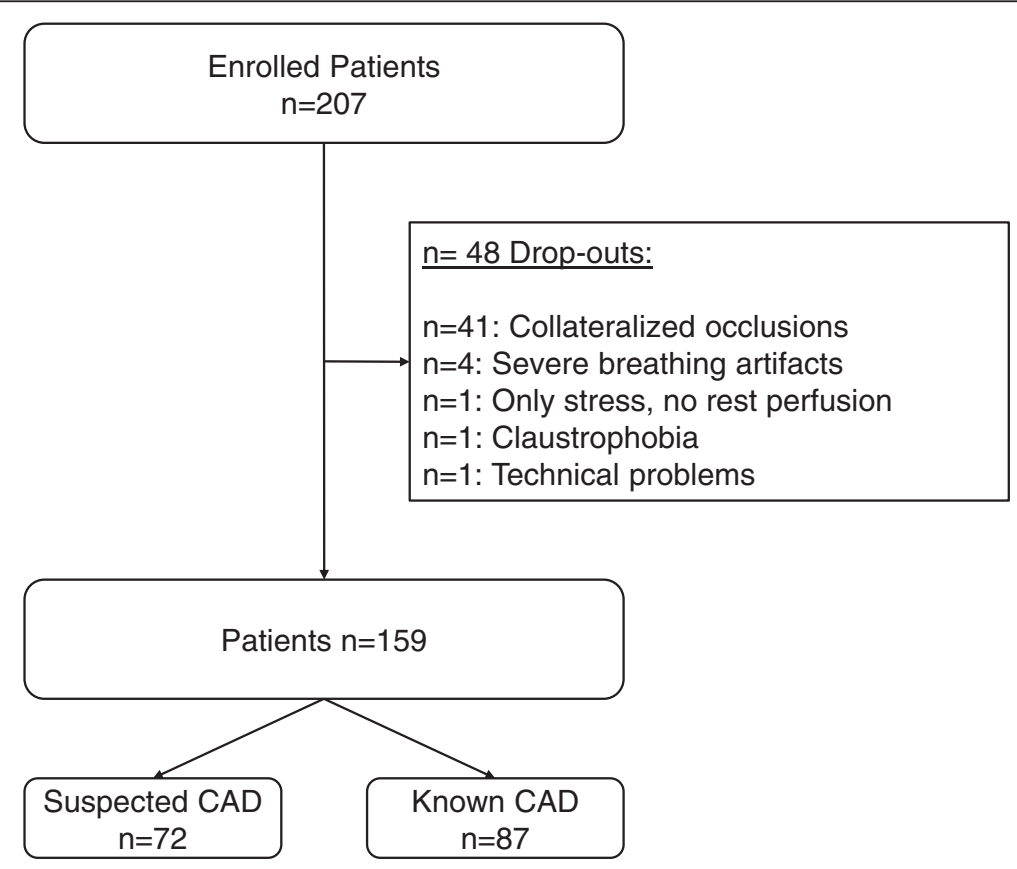

Fig. 1 Flow chart demonstrating the study population 
Table 1 Baseline characteristics

\begin{tabular}{|c|c|c|c|c|}
\hline & $\begin{array}{l}\text { Entire group } \\
(n=159)\end{array}$ & $\begin{array}{l}\text { Suspected CAD } \\
(n=72)\end{array}$ & $\begin{array}{l}\text { Known CAD } \\
(n=87)\end{array}$ & $p$ \\
\hline Age (yrs) & $71.1 \pm 10$ & $69.9 \pm 10.4$ & $72.2 \pm 9.6$ & 0.17 \\
\hline Gender (female) & $55(35 \%)$ & $39(54 \%)$ & $16(18 \%)$ & $<0.05$ \\
\hline \multicolumn{5}{|l|}{ CAD Risk Factors } \\
\hline Diabetes & $51(32 \%)$ & $20(28 \%)$ & $31(36 \%)$ & 0.31 \\
\hline Hypertension & $127(80 \%)$ & $58(81 \%)$ & $69(79 \%)$ & 1 \\
\hline Smoking ${ }^{a}$ & 49 (31\%) & $18(25 \%)$ & $31(36 \%)$ & 0.17 \\
\hline Hyperlipidemia & $105(66 \%)$ & $39(54 \%)$ & $66(76 \%)$ & 0.004 \\
\hline Family history of CVD & $55(35 \%)$ & $24(33 \%)$ & $31(36 \%)$ & 0.74 \\
\hline Menopause $^{b}$ & $52(95 \%)^{b}$ & $37(95 \%)^{b}$ & $15(94 \%)^{b}$ & 1 \\
\hline Obesity (BMI $\geq 30 \mathrm{~kg} / \mathrm{m}^{2}$ ) & $30(19 \%)$ & $17(24 \%)$ & $13(15 \%)$ & 0.15 \\
\hline Number of risk factors & $2.9 \pm 1.2$ & $2.9 \pm 1.3$ & $3.0 \pm 1.2$ & 0.53 \\
\hline \multicolumn{5}{|l|}{ Cardiac Arrhythmia } \\
\hline Heart rate at rest (beats/min.) & $67[60-78]$ & $68[60-80]$ & 66 [59-78] & 0.71 \\
\hline Heart rate at stress (beats/min.) & 85 [77-99] & 85 [78-101] & 85 [77-98] & 0.89 \\
\hline Atrial fibrillation & $64(40 \%)$ & $32(44 \%)$ & $32(37 \%)$ & 0.34 \\
\hline VES & $87(55 \%)$ & $35(49 \%)$ & $52(60 \%)$ & 0.20 \\
\hline Couplets & $15(9 \%)$ & $4(6 \%)$ & $11(13 \%)$ & 0.18 \\
\hline Triplets & $6(4 \%)$ & $2(3 \%)$ & $4(5 \%)$ & 0.69 \\
\hline Bigeminus & $32(20 \%)$ & $12(17 \%)$ & $20(23 \%)$ & 0.43 \\
\hline Trigeminus & $6(4 \%)$ & $3(4 \%)$ & $3(3 \%)$ & 1 \\
\hline SVES & $8(5 \%)$ & $5(7 \%)$ & $3(3 \%)$ & 0.47 \\
\hline \multicolumn{5}{|l|}{ Medication } \\
\hline Statins & $92(58 \%)$ & $34(47 \%)$ & $58(67 \%)$ & 0.03 \\
\hline Beta-blockers & $100(63 \%)$ & $38(53 \%)$ & $62(71 \%)$ & 0.03 \\
\hline Aspirin & $93(59 \%)$ & $34(47 \%)$ & $59(68 \%)$ & 0.02 \\
\hline ARB & $105(66 \%)$ & $41(57 \%)$ & $64(74 \%)$ & 0.06 \\
\hline Nitrates & 37 (23 \%) & $12(17 \%)$ & $25(29 \%)$ & 0.13 \\
\hline Diuretics & 77 (48 \%) & $31(43 \%)$ & $46(53 \%)$ & 0.33 \\
\hline \multicolumn{5}{|l|}{ Symptoms (multiple possible) } \\
\hline Chest pain & $107(67 \%)$ & $43(60 \%)$ & $64(74 \%)$ & 0.43 \\
\hline Dyspnea & $87(55 \%)$ & $46(64 \%)$ & $41(47 \%)$ & 0.04 \\
\hline Palpitations & $16(10 \%)$ & $9(13 \%)$ & $7(8 \%)$ & 0.09 \\
\hline Syncope & $10(6 \%)$ & $5(7 \%)$ & $5(6 \%)$ & 0.75 \\
\hline Reduced LV-EF & $56(35 \%)$ & $23(32 \%)$ & $33(38 \%)$ & 0.61 \\
\hline ECG abnormality & $105(66 \%)$ & $37(51 \%)$ & 68 (78 \%) & $<0.001$ \\
\hline Wall motion abnormality & 46 (29 \%) & 12 (17 \%) & $34(39 \%)$ & 0.02 \\
\hline
\end{tabular}

Values are $\mathrm{n}(\%)$, mean $\pm \mathrm{SD}$ or median [IQR]

suspected CAD CMR work-up of suspected CAD in patients without history of CAD, known CAD CMR work-up of ischemia in patients with prior myocardial infarction and/or revascularization procedure ( $\mathrm{PCl}$ or $\mathrm{CABG}), C A D$ coronary artery disease, $P C l$ percutaneous coronary intervention, $C A B G$ coronary artery bypass graft, $C V D$ cardiovascular disease, BMI body mass index, VES ventricular extrasystoles, SVES supraventricular extrasystoles, ARB angiotensin receptor blocker, CMR cardiac magnetic resonance, $L V$-EF left ventricular ejection fraction, ECG electrocardiography

${ }^{a}$ Current or ever-smokers

${ }^{\mathrm{b}}$ Calculated for females 
Arrhythmia was defined as atrial fibrillation, and/or frequent ectopic beats $>20 / \mathrm{min}$ (of ventricular or supraventricular origin) [13]. All arrhythmias were detected by ECG and/or Holter ECG, and had to be present during both adenosine stress and rest perfusion.

Analysis per coronary territory was made on a 17 segment model basis according to AHA guidelines as previously described [16]. Left coronary artery (LCA) included the following coronary arteries: left main (LM), left anterior descending (LAD) and left circumflex artery (LCX).

\section{CMR protocol}

Electrocardiogram (ECG) gated CMR imaging was performed in breath-hold using a $1.5 \mathrm{~T}$ Magnetom Aera (Siemens-Healthcare, Germany) in line with recommendations of the Society of Cardiac Magnetic Resonance, and the European Society of Cardiology Working Group EuroCMR, respectively [17].

Details of the CMR protocol have been reported previously [18]. In brief, steady-state free-precession cine images for assessment of LV function were acquired in multiple short-axis (every $10 \mathrm{~mm}$ throughout the LV) and three long-axis views. Adenosine $\left(140 \mu \mathrm{g} \cdot \mathrm{kg}^{-1} \cdot \mathrm{min}^{-1}\right)$ was infused under continuous electrocardiography and blood pressure monitoring for approximately $3 \mathrm{~min}$. At $2.5 \mathrm{~min}$ into the infusion, gadolinium $(0.07 \mathrm{mmol} / \mathrm{kg}$ Gadodiamide or Gadopentetate-Dimeglumine) first-pass imaging for assessment of stress perfusion was performed in three short axis views (basal, mid, apical, matched to cine locations excluding most basal and apical slices) using a saturation-recovery, single-shot, gradient-echo sequence ( $90^{\circ}$ pre-pulse before each slice; echo time, $1.1 \mathrm{~ms}$; delay time, $85-100 \mathrm{~ms}$; temporal resolution, $110-125 \mathrm{~ms}$; voxel size, $3.0 \times 1.8 \times 8.0 \mathrm{~mm}$ ). In order to speed up imaging parallel imaging with 2-fold acceleration was employed. Repeat first-pass images without adenosine 15 min later were performed for assessment of rest perfusion. Five minutes after rest perfusion (additional $0.07 \mathrm{mmol} / \mathrm{kg}$ Gadodiamide or Gadopentetate-Dimeglumine), late gadolinium enhancement was performed using a segmented inversionrecovery technique in the identical views as cine-CMR. The image acquisition protocol was completed in about $45 \mathrm{~min}$.

\section{CMR analysis}

Scans were analyzed by consensus of two experienced observers (S.G., H.M.), who were blinded to patient identity, clinical information, and the angiography results. A perfusion defect was defined as a regional dark area, that 1) persisted for $>2$ beats while other regions enhanced during the first-pass of contrast through the myocardium, and 2) involved the subendocardium $[19,20]$. Dark rim artifact was not regarded as perfusion deficit using previously described criteria [21].
Cine and contrast images were evaluated as described elsewhere [22]. In brief, endocardial and epicardial borders were outlined on the short axis cine images. Volumes and ejection fraction were derived by summation of epicardial and endocardial contours.

In patients referred for work-up of suspected CAD the Duke algorithm was used for diagnosis of CAD [18]: Patients were diagnosed having relevant stenosis/CAD if they had 1) evidence of LGE consistent with a prior myocardial infarction, or 2) no evidence of prior myocardial infarction, but perfusion defects present with adenosine that were absent or reduced at rest (reversible perfusion defect). If patients showed a matched perfusion defect under stress and rest perfusion, patients were considered having no relevant stenosis/CAD [18].

In patients referred for work-up of ischemia in known CAD two different algorithms were used depending on the presence of ischemic scar: 1) In the absence of ischemic scar on LGE images relevant stenosis/ischemia was defined as the presence of a reversible perfusion defect as described above [18]. 2) In the presence of ischemic scar on LGE images ischemia was defined as a mismatch between the first-pass stress perfusion defect and enhancement seen on LGE sequences, whereas a match between the first-pass stress perfusion defect and LGE was considered as chronic myocardial infarction with no additional reversible ischemia [23].

\section{Coronary angiography and analysis by coronary artery territory}

Coronary angiography was performed by standard techniques [24] and analyzed masked to identity, clinical information, and CMR results. Significant CAD was defined as $\geq 70 \%$ narrowing of the luminal diameter in at least one projection of at least one major epicardial artery, or $\geq 50 \%$ narrowing of the left main [2]. Native vessels with a diameter smaller than $2 \mathrm{~mm}$ were excluded from the analysis. Two experienced interventional cardiologists (S.G, H.M.) blinded to the results of the CMR imaging visually evaluated the coronary angiograms by consensus.

\section{Statistical analysis}

Absolute numbers and percentages were computed to describe the patient population. All continuous variables were tested for normality. Normally distributed continuous variables were expressed as means (with standard deviation) and skewed variables were presented as medians (with quartiles). Comparisons between groups were made using the Mann-Whitney $U$ test or the Fisher's exact test, as appropriate. Statistical tests were two-tailed; $p$ value $<0.05$ was considered significant. Positive and negative likelihood ratios (LR) were calculated. All statistical 
analyses were performed using SPSS, version 22.0 (IBM Corp., Armonk, NY, USA).

\section{Results}

\section{Patient characteristics}

In total 159 patients were included in the final analysis (Fig. 1). Table 1 summarizes the patient characteristics. At inclusion, patients were $71 \pm 10$ years of age and predominantly male (65\%). Atrial fibrillation was present in 64 patients (40\%), 87 patients (55\%) suffered from frequent ventricular extrasystoles (VES), and 8 patients (5\%) showed frequent supraventricular extrasystoles (SVES). The majority $(67 \%)$ had chest pain as primary reason to suspect significant $\mathrm{CAD}$, followed by dyspnea (55\%), and palpitations (10\%).

Seventy-two patients were referred for work-up of suspected CAD, and 87 patients were referred for work-up of ischemia in known CAD. The group with known CAD was older $(72.2 \pm 9.6)$ with fewer females (18 \%) than the group with suspected CAD (age 69.9 \pm 10.4 , $p=0.17 ; 54 \%$ females, $p<0.05)$. Patients presenting with known CAD had a higher prevalence of hyperlipidemia $(p=0.004)$, and wall motion abnormalities $(p=0.02)$ than patients with suspected CAD. Of note, the prevalence of the different types of arrhythmia was similar between the two groups.

\section{General CMR findings}

General CMR results are displayed in Table 2. Left ventricular ejection fraction (LV-EF) in the study population was mildly impaired (median 54\%) with normal mean cardiac volumes. Overall, CMR perfusion revealed LCA ischemia in $31 \%$, and RCA ischemia in $23 \%$ of patients. CAD-type LGE was present in $47 \%$ of patients.

Table 2 CMR results

\begin{tabular}{lllll}
\hline Parameter & $\begin{array}{l}\text { Entire group } \\
(n=159)\end{array}$ & $\begin{array}{l}\text { Suspected CAD } \\
(n=72)\end{array}$ & $\begin{array}{l}\text { Known CAD } \\
(n=87)\end{array}$ & $p$ \\
\hline LV-EF $(\%)$ & $54[39-66]$ & $61[45-67]$ & $50[34-64]$ & 0.04 \\
LV-EDV $(\mathrm{ml})$ & $124[101-168]$ & $116[91-145]$ & $133[106-181]$ & 0.01 \\
LV-ESV $(\mathrm{ml})$ & $54[32-98]$ & $49[28-69]$ & $68[40-109]$ & 0.007 \\
LA (cm $\left.{ }^{2}\right)$ & $26[21-35]$ & $27[22-38]$ & $26[21-34]$ & 0.43 \\
IVS $(\mathrm{mm})$ & $12[10-13]$ & $11[10-13]$ & $12[10-14]$ & 0.58 \\
Ischemia LCA & $49(31 \%)$ & $13(18 \%)$ & $36(41 \%)$ & 0.001 \\
Ischemia RCA & $36(23 \%)$ & $7(10 \%)$ & $29(33 \%)$ & $<0.001$ \\
CAD-type LGE & $74(47 \%)$ & $15(21 \%)$ & $59(68 \%)$ & $<0.001$
\end{tabular}

Values are median [IQR], or $\mathrm{n}(\%)$

$C A D$ coronary artery disease, suspected CAD CMR work-up of suspected CAD in patients without history of CAD, known CAD CMR work-up of ischemia in patients with prior myocardial infarction and/or revascularization procedure $(\mathrm{PCl}$ or $C A B G), L V$ left ventricular, $E F$ ejection fraction, $E D V$ end-diastolic volume, ESV end-systolic volume, $L A$ left atrium, IVS interventricular septum, $L C A L M+L A D+$ $L C X, L C A$ left coronary artery, $L M$ left main, $L A D$ left anterior descending, $L C X$ left circumflex artery, $L G E$ late gadolinium enhancement
Among patients with known CAD, LV-EF was significantly lower compared to the suspected CAD group, $p=0.04$. Conversely, left ventricular end-diastolic volumes (LV-EDV) and left ventricular end-systolic volumes (LV-ESV) were significantly larger in the known CAD group (LV-EDV $p=0.01$, LV-ESV $p=0.007$ respectively). In addition, patients with known CAD were diagnosed with relevant stenosis/ischemia more frequently than patients with suspected CAD $(p=0.001, p<0.001$, respectively). CAD-type LGE was also more common in the known CAD group (68 vs. $21 \%, p<0.001$ ).

\section{Diagnostic performance of CMR Entire population}

Overall diagnostic accuracy of adenosine stress CMR for the detection of $\geq 70 \%$ stenosis on coronary angiography was $73 \%$ (sensitivity $72 \%$, specificity $76 \%$ ) for patients with suspected or known CAD (Table 3). On a per coronary territory basis the diagnostic accuracy of CMR was $77 \%$ for LCA stenosis (sensitivity $78 \%$, specificity $77 \%$ ) and $82 \%$ for detection of RCA stenosis (sensitivity $63 \%$, specificity $88 \%$ ).

Table 3 Diagnostic performance of CMR stress testing for the detection of $\geq 70 \%$ stenosis on coronary angiography in all patients $(n=159)$

\begin{tabular}{|c|c|c|c|}
\hline & Per patient & $\mathrm{LCA}^{a}$ & RCA \\
\hline \multicolumn{4}{|c|}{ All Types of Arrhythmiab } \\
\hline Sensitivity & $72 \%(49 / 68)$ & 78 \% (53/68) & $63 \%(24 / 38)$ \\
\hline Specificity & $76 \%(69 / 91)$ & $77 \%(70 / 91)$ & $88 \%(107 / 121)$ \\
\hline $\begin{array}{l}\text { Diagnostic } \\
\text { Accuracy }\end{array}$ & $73 \%(118 / 159)$ & $77 \%(123 / 159)$ & $82 \%(131 / 159)$ \\
\hline $\mathrm{LR}+$ & 3.00 & 3.39 & 5.25 \\
\hline LR- & 0.37 & 0.29 & 0.42 \\
\hline \multicolumn{4}{|l|}{ AFib Only } \\
\hline Sensitivity & $71 \%(25 / 35)$ & $81 \%(22 / 27)$ & $63 \%(10 / 16)$ \\
\hline Specificity & $69 \%(20 / 29)$ & $76 \%(28 / 37)$ & $88 \%(42 / 48)$ \\
\hline $\begin{array}{l}\text { Diagnostic } \\
\text { Accuracy }\end{array}$ & $70 \%(45 / 64)$ & $78 \%(50 / 64)$ & $81 \%(52 / 64)$ \\
\hline LR+ & 2.29 & 3.38 & 5.25 \\
\hline LR- & 0.42 & 0.25 & 0.42 \\
\hline \multicolumn{4}{|l|}{ VES Only } \\
\hline Sensitivity & $74 \%(23 / 31)$ & $75 \%(30 / 40)$ & $65 \%(13 / 20)$ \\
\hline Specificity & $82 \%(46 / 56)$ & $81 \%(38 / 47)$ & $90 \%(61 / 67)$ \\
\hline $\begin{array}{l}\text { Diagnostic } \\
\text { Accuracy }\end{array}$ & $79 \%(69 / 87)$ & $78 \%(68 / 87)$ & $85 \%(74 / 87)$ \\
\hline $\mathrm{LR}+$ & 4.11 & 3.95 & 6.05 \\
\hline LR- & 0.32 & 0.31 & 0.39 \\
\hline
\end{tabular}

\section{Values are $\%(n)$}

AFib atrial fibrillation, VES ventricular extrasystoles, SVES supraventricular extrasystoles, $L R+$ positive likelihood ratio, $L R$ - negative likelihood ratio ${ }^{a} \mathrm{LCA}=\mathrm{LM}+\mathrm{LAD}+\mathrm{LCX}$, abbreviations see Table 2

${ }^{\mathrm{b}}$ All types of arrhythmia: $n=64 \mathrm{AFib}+n=87 \mathrm{VES}+n=8$ SVES 
Looking at patients presenting with atrial fibrillation $(n=64)$ revealed a diagnostic accuracy of $70 \%$ for CMR (sensitivity $71 \%$, specificity $69 \%$ ), which is lower than in the 87 patients presenting with VES (diagnostic accuracy $79 \%$, sensitivity $74 \%$, specificity $82 \%)$. On a per coronary territory basis, the diagnostic accuracy for detection of LCA and RCA stenosis was good in patients with atrial fibrillation $(78 \%, 81 \%$ respectively), and in patients with VES (78 \%, $85 \%$ respectively).

Considering the low number of patients with SVES $(n=8)$, these patients were included in the entire population analysis. Five of those patients were in the suspected CAD group, and three out of five were classified correctly as negative by CMR. The other three patients had known CAD, two of them had coronary stenosis on coronary angiography, one of them was correctly identified by CMR.

\section{Patients with suspected CAD}

Diagnostic accuracy of CMR stress testing for the detection of $\geq 70 \%$ stenosis in patients with suspected CAD was $75 \%$ (sensitivity $80 \%$, specificity $74 \%$ ), positive likelihood ratio (LR) 3.08, negative LR 0.27 (Table 4). The prevalence of significant coronary stenosis on coronary angiography was $14 \%$ (10 out of 72 patients with suspected CAD).

CMR identified $80 \%$ of the patients with suspected CAD and stenosis of the LCA correctly, yielding to a diagnostic accuracy of $76 \%$ (specificity $76 \%$ ), positive LR 3.33 and negative LR 0.26. For the RCA, CMR revealed a diagnostic accuracy of $89 \%$, with a sensitivity of $100 \%$, and a specificity of $89 \%$, positive LR 8.33, negative LR 0 . Figure 2 demonstrates two typical patients with suspected CAD and different types of arrhythmia.

\section{Patients with known CAD}

The diagnostic accuracy of CMR stress for detection of $\geq 70 \%$ stenosis in patients with known CAD was $74 \%$ (sensitivity $71 \%$, specificity $79 \%$ ), positive LR 3.38,

Table 4 Diagnostic performance of CMR stress testing for the detection of $\geq 70 \%$ stenosis on coronary angiography in patients with suspected CAD by use of the Duke algorithm ${ }^{a}$

\begin{tabular}{llll}
\hline & Per patient & LCA $^{\text {b }}$ & RCA \\
\hline Sensitivity & $80 \%(8 / 10)$ & $80 \%(8 / 10)$ & $100 \%(1 / 1)$ \\
Specificity & $74 \%(46 / 62)$ & $76 \%(47 / 62)$ & $89 \%(63 / 71)$ \\
Diagnostic Accuracy & $75 \%(54 / 72)$ & $76 \%(55 / 72)$ & $89 \%(64 / 72)$ \\
LR+ & 3.08 & 3.33 & 8.33 \\
LR- & 0.27 & 0.26 & 0 \\
\hline
\end{tabular}

Values are \% (n)

suspected $C A D$ CMR work-up of suspected CAD in patients without history of CAD

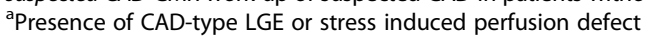

${ }^{\mathrm{b}} \mathrm{LCA}=\mathrm{LM}+\mathrm{LAD}+\mathrm{LCX} ; \mathrm{n}=32 \mathrm{AFib}+\mathrm{n}=35 \mathrm{VES}+n=5$ SVES; other abbreviations see Table 3 negative LR 0.37 , see Table 5 . The prevalence of significant coronary stenosis on coronary angiography was $67 \%$ (58 out of 87 patients with known CAD).

CMR identified $78 \%$ of patients with known CAD and stenosis $\geq 70 \%$ in the LCA correctly, yielding a diagnostic accuracy of $78 \%$ and a specificity of $79 \%$, positive LR 3.71, and negative LR 0.28. For the RCA, CMR revealed a diagnostic accuracy of $77 \%$, with a sensitivity of $62 \%$, and a specificity of $88 \%$, positive LR 5.17, negative LR 0.43. Typical CMR results are displayed in Fig. 3

\section{Discussion}

To the best of our knowledge, this is the first study evaluating the diagnostic performance of adenosine stress CMR for detection of significant coronary stenosis in patients with different types of arrhythmia. Our data indicate that adenosine stress CMR performs well for detection of relevant coronary stenosis in patients with suspected CAD (diagnostic accuracy $75 \%$ ), and also in patients with known CAD (diagnostic accuracy $74 \%$ ), despite the presence of various arrhythmias during the CMR procedure. These results underscore the increasing value of adenosine stress CMR in the real world clinical routine.

\section{Patient characteristics}

The average patient age and gender distribution are similar to previous stress CMR studies in which patients with arrhythmia where usually excluded from analysis [5]. This also holds true for the clinical symptoms leading to CMR referral in the present population [25]. Types of arrhythmia found in our patients include atrial fibrillation, frequent VES and frequent SVES, which are known to be associated with CAD [8-10]. As to expect, the subgroup with known CAD was older (72.2 \pm 9.6 years), with fewer females (18 \%) and higher prevalence of hyperlipidemia than the group with suspected CAD $(69.9 \pm 10.4$ years, $p=0.17 ; 54 \%$ females, $p<0.05)$.

\section{General CMR findings}

Median LV-EF of all patients was $54 \%$, which is comparable to other studies evaluating the diagnostic performance of adenosine stress CMR in a mixed patient population comprising patients with suspected and known CAD [23]. Patients with known CAD had a lower LV-EF and larger end-diastolic and end-systolic volumes, most likely explained by the higher prevalence of ischemic scar represented by CAD-type LGE (68 vs. $21 \%$ in the suspected CAD group), resulting in reduced LV-EF and ventricular remodeling. Ischemia was also more common in patients with known CAD than in the group with suspected $\mathrm{CAD}$, respectively. 

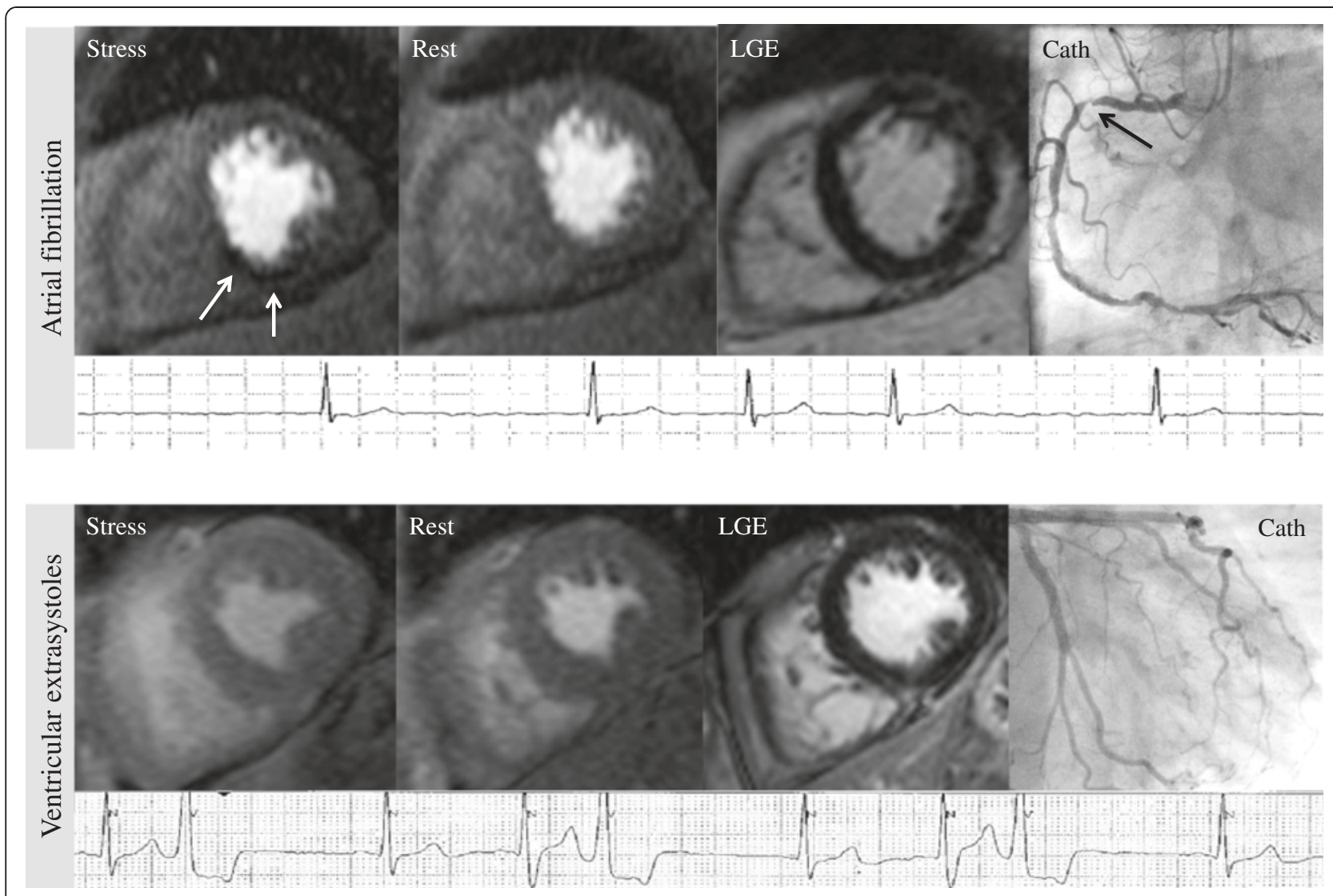

Fig. 2 Patients with suspected CAD, but different types of arrhythmia: Top row: 72-year old male with atrial fibrillation presenting for work-up of suspected CAD. CMR revealed no LGE, but a reversible perfusion defect at the inferoseptal wall (white arrows), highly suggestive of significant RCA stenosis. Coronary angiography revealed high-grade RCA stenosis (black arrow). Bottom row: 69-year old female with frequent VES and atypical chest pain presenting for work-up of suspected CAD. LGE was negative, stress perfusion revealed no perfusion defect, resulting in the CMR diagnosis "no CAD". Coronary angiography confirmed unobstructed coronary arteries

\section{Diagnostic performance of CMR Entire population}

Looking at the entire population, the diagnostic accuracy of stress CMR for the detection of $\geq 70 \%$ stenosis on coronary angiography was $73 \%$ (sensitivity $72 \%$, specificity $76 \%$ ) for all 159 patients with suspected or known

Table 5 Diagnostic performance of CMR stress testing for the detection of $\geq 70 \%$ stenosis on coronary angiography in patients with known CAD

\begin{tabular}{llll}
\hline & Per patient & LCA $^{\text {a }}$ & RCA \\
\hline Sensitivity & $71 \%(41 / 58)$ & $78 \%(45 / 58)$ & $62 \%(23 / 37)$ \\
Specificity & $79 \%(23 / 29)$ & $79 \%(23 / 29)$ & $88 \%(44 / 50)$ \\
Diagnostic Accuracy & $74 \%(64 / 87)$ & $78 \%(68 / 87)$ & $77 \%(67 / 87)$ \\
LR+ & 3.38 & 3.71 & 5.17 \\
LR- & 0.37 & 0.28 & 0.43 \\
\hline
\end{tabular}

Values are $\%(n)$

known CAD CMR work-up of ischemia in patients with prior myocardial infarction and/or revascularization procedure ( $\mathrm{PCl}$ or $\mathrm{CABG})$

${ }^{\mathrm{a}} \mathrm{LCA}=\mathrm{LM}+\mathrm{LAD}+\mathrm{LCX}, n=32 \mathrm{AFib}+n=52 \mathrm{VES}+n=3$ SVES, abbreviations see Table 3
CAD, and different types of arrhythmia. This is lower than reported in a large meta-analysis [5] calculating a sensitivity of $90 \%$ and a specificity of $81 \%$. However, many studies of this meta-analysis excluded patients with arrhythmia to improve image quality, which most likely explains the difference to our data based on patients presenting with arrhythmia only.

Analyzing our entire population data per coronary territory demonstrate CMR to yield a diagnostic accuracy of $77 \%$ for the LCA (sensitivity $78 \%$, specificity $77 \%$ ), and of $82 \%$ for the RCA (sensitivity $63 \%$, specificity $88 \%$ ). This is also lower than in the meta-analysis mentioned above, demonstrating sensitivities of 83,76 and $78 \%$ and specificities of 83,87 , and $87 \%$ for LAD, LCX and RCA, respectively. However, patients with arrhythmias were excluded in most studies of this meta-analysis [5].

Comparing patients with atrial fibrillation to patients with VES (Table 3) reveals a good diagnostic accuracy for LCA and RCA (78 and $81 \%$ ) in the atrial fibrillation group, and in patients with VES (78 and $85 \%$ ). However, the sensitivities for detecting relevant RCA stenosis were 

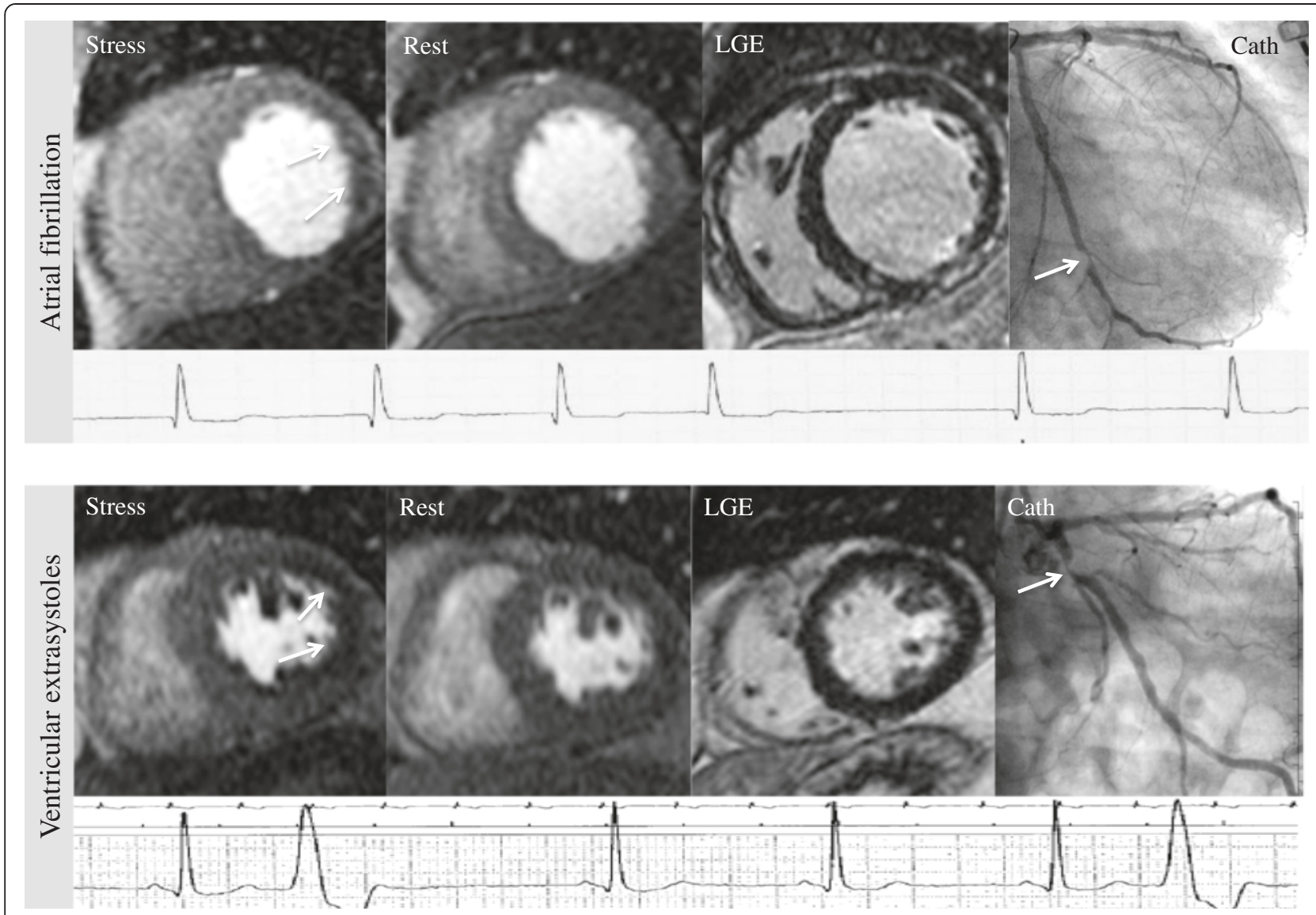

Fig. 3 Patients with known CAD. Top row: 71-year old female with atrial fibrillation and known CAD (myocardial infarction two years ago) presented for work-up of new ischemia. LGE revealed a transmural infarction of the inferior wall. Stress perfusion demonstrated a reversible perfusion defect of the lateral wall (white arrows), highly suggestive of significant LCX stenosis. This could be confirmed by coronary angiography: LCX had a high-grade proximal stenosis (white arrow), RCA showed coronary plaques, but no significant stenosis. Bottom row: 73-year old male with typical angina, frequent VES, and known CAD (prior stenosis of the LAD, in which PCI was performed 12 years ago). LGE revealed no scar, but stress perfusion demonstrated a large perfusion defect in the lateral wall, suggestive of LCX stenosis. On coronary angiography, severe LCX stenosis could be confirmed

lower in both groups when compared to the LCA (atrial fibrillation 63 vs. $81 \%$, VES 65 vs. $75 \%$ ). This is in accordance with other studies [5], reporting a higher sensitivity for detection of stenosis in the LAD when compared to LCX and RCA, most likely due to the surface radiofrequency coil receiving lower signal intensities from the inferior and lateral segments [5].

\section{Patients with suspected CAD}

Evaluating the subgroup presenting for work-up of suspected CAD the diagnostic accuracy was $75 \%$ (sensitivity $80 \%$, specificity $74 \%$ ). This is less than reported by Klem et al. [18], who first described the Duke algorithm for work-up of suspected CAD by combining LGE and perfusion sequences in 92 patients. However, in contrast to the present data, the Klem population only included one patient with atrial fibrillation and two patients with VES.
Among our 72 patients with suspected CAD the prevalence of significant coronary stenosis was low (14\%), underscoring the need of noninvasive imaging in patients with arrhythmia before undergoing coronary angiography. One reason for the low prevalence of CAD might be that patients with arrhythmia are at an "increased risk" to be referred to coronary angiography due to previous inconclusive exercise tests, or the presence of arrhythmia itself in combination with risk factors and complaints. Interestingly, this finding nicely matches the results of Smit et al. who performed a myocardial perfusion single photon emission computed tomography (SPECT) analysis in patients with atrial fibrillation and suspected CAD. The prevalence of CAD ( $\geq 70 \%$ stenosis) in this group was $13 \%$ [26].

In a per coronary territory analysis, the diagnostic accuracy for the LCA was $76 \%$ (sensitivity $80 \%$, specificity 
$76 \%)$. For the RCA our data revealed a diagnostic accuracy of $89 \%$ (sensitivity $100 \%$, specificity $89 \%$ ), completely in line with the results of Klem et al. [18]. However, it must be noted that only one patient with suspected CAD had $\geq 70 \%$ RCA stenosis.

\section{Patients with known CAD}

Focusing on the subgroup with known CAD the diagnostic accuracy of CMR stress testing for the detection of $\geq 70 \%$ stenosis was $74 \%$ (sensitivity $71 \%$, specificity $79 \%$ ). A study by Klein et al. investigated the diagnostic performance of adenosine perfusion CMR in 78 patients [27] reporting a diagnostic accuracy of $82 \%$ for detection of $\geq 50 \%$ stenosis (sensitivity $77 \%$, specificity $90 \%$ ), excluding patients with atrial fibrillation.

Per coronary territory the diagnostic accuracy for the LCA was 78 and $77 \%$ for the RCA, which is also quite in line with other reports [23, 27]. Figure 4 demonstrates the feasibility of adenosine stress CMR in a patient presenting with frequent VES (bigeminus).

\section{Limitations}

Limitations of the present study are, that adenosine stress CMR was compared with invasive coronary angiography, which is not the perfect gold standard for comparison as functional significance of coronary obstruction and luminal diameter stenosis are known to show only moderate correlation. Furthermore, it is important to keep in mind that the algorithm used for CMR analysis of patients with suspected CAD is intended to detect significant obstruction of the epicardial coronaries compared to invasive coronary angiography ( $>70 \%$ stenosis). Thus, perfusion defects that were considered as artifacts according to the algorithm used in this analysis may be a surrogate parameter for microvascular dysfunction. Hence, it might be possible that these patients who suffer partly from distinct anginal symptoms were classified as healthy by CMR, which could be confirmed by coronary angiography. Another limitation is the selection bias introduced by excluding patients with collateralized occlusions and CMR studies with severe breathing artifacts. Despite introducing a bias, we believe that the exclusion of collateralized occlusions is favorable in order to keep the data consistent, since in those cases no ischemia may be present despite occlusion of the vessel. Furthermore, removal of CMR data sets due to severe breathing artifacts seems reasonable since aim of our study was to evaluate the impact of arrhythmia (and not of severe breathing artifacts) on the diagnostic accuracy of an adenosine stress CMR test. Moreover, it should be stated that only a few patients (4 out of 163 patients, $=0.02 \%$ ) were excluded due to severe breathing artifacts.

\section{Clinical implications}

On the basis of the data presented it may be safe to assume that the diagnostic performance of adenosine stress CMR for detection of significant coronary stenosis is somewhat impaired in patients presenting with

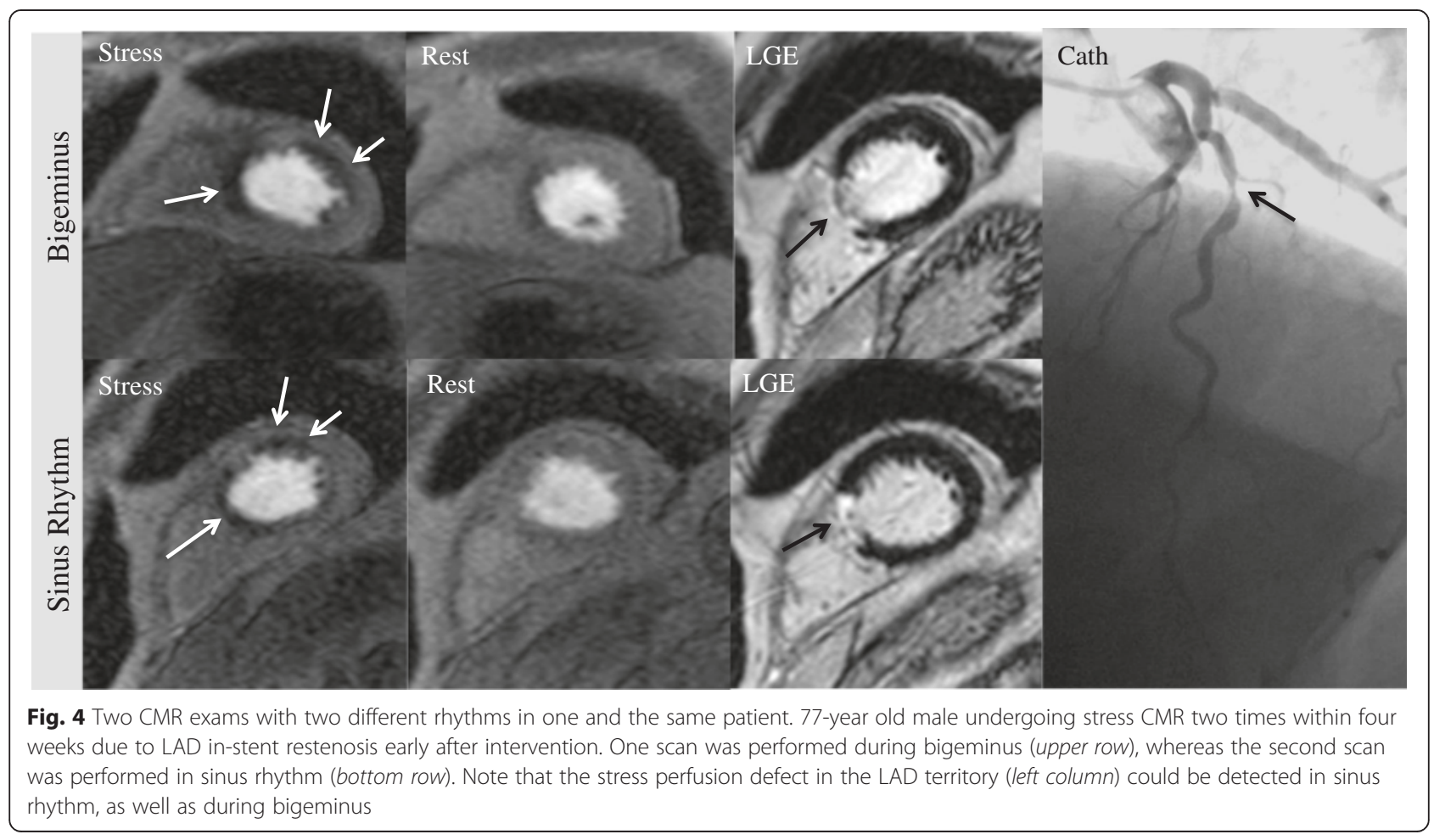


arrhythmias compared to patients without arrhythmias (75 vs. $88 \%$ for suspected CAD [18] and 74 vs. $82 \%$ in known CAD [27]), but still sufficient for clinical routine use. In fact, with a sensitivity of $72 \%$, and specificity of $76 \%$ (overall diagnostic accuracy of $73 \%$ ) adenosine stress CMR in patients with arrhythmias performs well in comparison to other stress testing modalities (SPECT: sensitivity 73-92 \%, specificity 63-87; stress echocardiography: sensitivity $80-85 \%$, specificity $80-88 \%$; exercise ECG: sensitivity 45-50 \%, specificity 85-90\%) [2], underscoring the value of adenosine stress CMR in a real world clinical setting.

Unfortunately, the current study was not designed to evaluate the prognostic value of stress CMR in patients with arrhythmias. However, given the encouraging results with regard to the diagnostic performance in those patients, and the results of the EuroCMR Registry demonstrating a good prognostic value of adenosine stress CMR in patients presenting for work-up of suspected CAD [28], including those with arrhythmias, it is likely that adenosine stress CMR also has a good prognostic value in arrhythmic patients. Nevertheless, additional data is needed to underscore this important point.

\section{Conclusions}

The present data demonstrates a good diagnostic performance of adenosine stress CMR for detection of significant coronary stenosis in patients with arrhythmia presenting for 1) work-up of suspected CAD, or 2) work-up of ischemia in known CAD. This holds true in the entire population for a per patient, as well as a per coronary territory analysis.

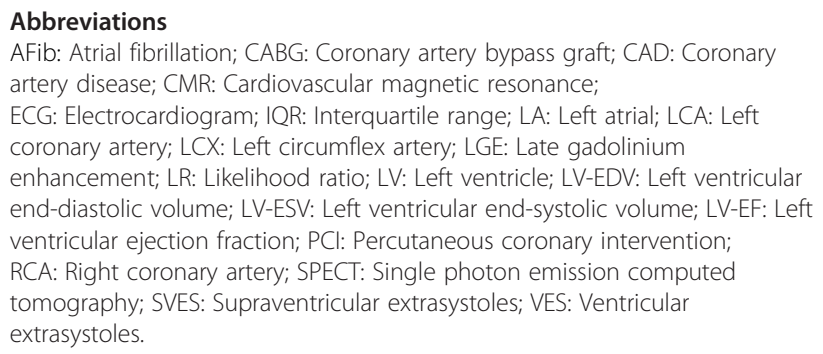

\section{Competing interests}

The authors declare that they have no competing interests.

\begin{abstract}
Authors'contributions
SG and HS contributed to the idea and design of the study, acquired and analyzed the data, and wrote the report. SB, SG, KB, US contributed to the idea and design of the study, analysis of the data, and revision of the report. HM designed the study, contributed to the acquisition and analysis of the data, and wrote the report. All authors read and approved the final manuscript.
\end{abstract}

\section{Funding sources}

This work was funded in part by the Robert Bosch Foundation 1) clinical research grant for CMR risk stratification in $\mathrm{HCM}$ and 2) clinical research grant for inflammatory heart disease KKF-11-18, KKF-13-2.
Received: 7 July 2015 Accepted: 21 October 2015

Published online: 05 November 2015

\section{References}

1. Lloyd-Jones D, Adams RJ, Brown TM, Carnethon M, Dai S, De Simone G, et al. Heart disease and stroke statistics-2010 update: a report from the American Heart Association. Circulation. 2010;121:e46-e215.

2. Montalescot G, Sechtem U, Achenbach S, Andreotti F, Arden C, Budaj A, et al. 2013 ESC guidelines on the management of stable coronary artery disease: the Task Force on the management of stable coronary artery disease of the European Society of Cardiology. Eur Heart J. 2013;34:2949-3003.

3. Windecker S, Kolh P, Alfonso F, Collet JP, Cremer J, Falk V, et al. 2014 ESC/ EACTS Guidelines on myocardial revascularization: The Task Force on Myocardial Revascularization of the European Society of Cardiology (ESC) and the European Association for Cardio-Thoracic Surgery (EACTS)Developed with the special contribution of the European Association of Percutaneous Cardiovascular Interventions (EAPCI). Eur Heart J. 2014;35:2541-619.

4. Nandalur KR, Dwamena BA, Choudhri AF, Nandalur MR, Carlos RC. Diagnostic performance of stress cardiac magnetic resonance imaging in the detection of coronary artery disease: a meta-analysis. J Am Coll Cardiol. 2007;50:1343-53.

5. Hamon M, Fau G, Nee G, Ehtisham J, Morello R, Hamon M. Meta-analysis of the diagnostic performance of stress perfusion cardiovascular magnetic resonance for detection of coronary artery disease. J Cardiovasc Magn Reson: Off J Soc Cardiovascular Magnetic Resonance. 2010;12:29.

6. Schwitter J, Wacker CM, Wilke N, Al-Saadi N, Sauer E, Huettle K, et al. MR-IMPACT II: Magnetic Resonance Imaging for Myocardial Perfusion Assessment in Coronary artery disease Trial: perfusion-cardiac magnetic resonance vs. single-photon emission computed tomography for the detection of coronary artery disease: a comparative multicentre, multivendor trial. Eur Heart J. 2013;34:775-81.

7. Greenwood JP, Maredia N, Younger JF, Brown JM, Nixon J, Everett CC, et al. Cardiovascular magnetic resonance and single-photon emission computed tomography for diagnosis of coronary heart disease (CE-MARC): a prospective trial. Lancet. 2012;379:453-60.

8. Bigger Jr JT, Dresdale FJ, Heissenbuttel RH, Weld FM, Wit AL. Ventricular arrhythmias in ischemic heart disease: mechanism, prevalence, significance, and management. Prog Cardiovasc Dis. 1977;19:255-300.

9. AFFIRM Investigator, Atrial Fibrillation Follow-up Investigation of Rhythm Management. Baseline characteristics of patients with atrial fibrillation: the AFFIRM Study. Am Heart J. 2002;143:991-1001.

10. Hohnloser SH, Crijns HJ, van Eickels M, Gaudin C, Page RL, Torp-Pedersen C, et al. Effect of dronedarone on cardiovascular events in atrial fibrillation. N Engl J Med. 2009;360:668-78.

11. Giang TH, Nanz D, Coulden R, Friedrich M, Graves M, Al-Saadi N, et al. Detection of coronary artery disease by magnetic resonance myocardial perfusion imaging with various contrast medium doses: first European multi-centre experience. Eur Heart J. 2004;25:1657-65.

12. Merkle N, Wohrle J, Grebe O, Nusser T, Kunze M, Kestler HA, et al. Assessment of myocardial perfusion for detection of coronary artery stenoses by steady-state, free-precession magnetic resonance first-pass imaging. Heart. 2007;93:1381-5.

13. Schwitter J, Wacker CM, van Rossum AC, Lombardi M, Al-Saadi N, Ahlstrom $\mathrm{H}$, et al. MR-IMPACT: comparison of perfusion-cardiac magnetic resonance with single-photon emission computed tomography for the detection of coronary artery disease in a multicentre, multivendor, randomized trial. Eur Heart J. 2008:29:480-9.

14. Kohli P, Waters DD. Looking for coronary disease in patients with atrial fibrillation. Can J Cardiol. 2014;30:861-3.

15. Mahrholdt H, Wagner A, Judd RM, Sechtem U, Kim RJ. Delayed enhancement cardiovascular magnetic resonance assessment of nonischaemic cardiomyopathies. Eur Heart J. 2005;26:1461-74.

16. Cerqueira MD, Weissman NJ, Dilsizian V, Jacobs AK, Kaul S, American Heart Association Writing Group on Myocardial Segmentation and Registration for Cardiac Imaging, et al. Standardized myocardial segmentation and nomenclature for tomographic imaging of the heart. A statement for healthcare professionals from the cardiac imaging committee of the council on clinical cardiology of the american heart association. Circulation. 2002;105(4):539-42. 
17. Kramer CM, Barkhausen J, Flamm SD, Kim RJ, Nagel E. Standardized cardiovascular magnetic resonance imaging (CMR) protocols, society for cardiovascular magnetic resonance: board of trustees task force on standardized protocols. J Cardiovasc Magn Reson: Off J Soc Cardiovascular Magnetic Resonance. 2008;10:35.

18. Klem I, Heitner JF, Shah DJ, Cawley P, Behar V, Weinsaft J, et al. Improved detection of coronary artery disease by stress perfusion cardiovascular magnetic resonance with the use of delayed enhancement infarction imaging. J Am Coll Cardiol. 2006;47:1630-8.

19. Greulich S, Bruder O, Parker M, Schumm J, Grün S, Schneider S, et al. Comparison of exercise electrocardiography and stress perfusion CMR for the detection of coronary artery disease in women. J Cardiovasc Magn Reson : Off J Soc Cardiovascular Magnetic Resonance. 2012;14:36.

20. Klem I, Greulich S, Heitner JF, Kim H, Vogelsberg H, Kispert EM, et al. Value of cardiovascular magnetic resonance stress perfusion testing for the detection of coronary artery disease in women. JACC Cardiovasc Imaging. 2008;1:436-45.

21. Di Bella EV, Parker DL, Sinusas AJ. On the dark rim artifact in dynamic contrast-enhanced MRI myocardial perfusion studies. Magn Reson Med: Off J Soc Magnetic Resonance Med Soc Magnetic Resonance Med. 2005:54:1295-9.

22. Mahrholdt H, Goedecke C, Wagner A, Meinhardt G, Athanasiadis A, Vogelsberg $\mathrm{H}$, et al. Cardiovascular magnetic resonance assessment of human myocarditis: a comparison to histology and molecular pathology. Circulation. 2004;109:1250-8.

23. Bernhardt P, Spiess J, Levenson B, Pilz G, Höfling B, Hombach V, et al. Combined assessment of myocardial perfusion and late gadolinium enhancement in patients after percutaneous coronary intervention or bypass grafts: a multicenter study of an integrated cardiovascular magnetic resonance protocol. JACC Cardiovasc Imaging. 2009;2:1292-300.

24. Scanlon PJ, Faxon DP, Audet AM, Carabello B, Dehmer GJ, Eagle KA, et al. ACC/AHA guidelines for coronary angiography: executive summary and recommendations. A report of the american college of cardiology/american heart association task force on practice guidelines (committee on coronary angiography) developed in collaboration with the society for cardiac angiography and interventions. Circulation. 1999;99:2345-57.

25. Bingham SE, Hachamovitch R. Incremental prognostic significance of combined cardiac magnetic resonance imaging, adenosine stress perfusion, delayed enhancement, and left ventricular function over preimaging information for the prediction of adverse events. Circulation. 2011;123:1509-18.

26. Smit MD, Tio RA, Slart RH, Zijlstra F, Van Gelder IC. Myocardial perfusion imaging does not adequately assess the risk of coronary artery disease in patients with atrial fibrillation. Europace. 2010;12:643-8.

27. Klein C, Nagel E, Gebker R, Kelle S, Schnackenburg B, Graf K, et al. Magnetic resonance adenosine perfusion imaging in patients after coronary artery bypass graft surgery. JACC Cardiovasc Imaging. 2009;2:437-45.

28. Bruder O, Wagner A, Lombardi M, Schwitter J, Pilz G, Nothnagel D, et al. European Cardiovascular Magnetic Resonance (EuroCMR) registry-multi national results from 57 centers in 15 countries. J Cardiovasc Magn Reson. 2013;15:9.

\section{Submit your next manuscript to BioMed Central and take full advantage of:}

- Convenient online submission

- Thorough peer review

- No space constraints or color figure charges

- Immediate publication on acceptance

- Inclusion in PubMed, CAS, Scopus and Google Scholar

- Research which is freely available for redistribution 